\title{
Simulating Dissolution of Intravitreal Triamcinolone Acetonide Suspensions in an Anatomically Accurate Rabbit Eye Model
}

\author{
Paul J. Missel • Marc Horner • R. Muralikrishnan
}

Received: 13 February 2010 / Accepted: 15 April 2010 / Published online: 14 May 2010

(C) The Author(s) 2010. This article is published with open access at Springerlink.com

\begin{abstract}
Purpose A computational fluid dynamics (CFD) study examined the impact of particle size on dissolution rate and residence of intravitreal suspension depots of Triamcinolone Acetonide (TAC). Methods A model for the rabbit eye was constructed using insights from high-resolution NMR imaging studies (Sawada 2002). The current model was compared to other published simulations in its ability to predict clearance of various intravitreally injected materials. Suspension depots were constructed explicitly rendering individual particles in various configurations: 4 or $16 \mathrm{mg}$ drug confined to a $100 \mu \mathrm{L}$ spherical depot, or $4 \mathrm{mg}$ exploded to fill the entire vitreous. Particle size was reduced systematically in each configuration. The convective diffusion/ dissolution process was simulated using a multiphase model.

Results Release rate became independent of particle diameter below a certain value. The size-independent limits occurred for particle diameters ranging from 77 to $428 \mu \mathrm{M}$ depending upon the depot configuration. Residence time predicted for the spherical depots in the size-independent limit was comparable to that observed in vivo.
\end{abstract}

Conclusions Since the size-independent limit was several-fold greater than the particle size of commercially available

P. J. Missel $(\bowtie)$

Drug Delivery, Alcon Research Ltd.

Mail Stop R2-45, 620I South Freeway

Fort Worth, Texas 76134, USA

e-mail: paul.missel@alconlabs.com

M. Horner

ANSYS, Inc.

Evanston, Illinois, USA

R. Muralikrishnan

ANSYS Fluent India Pvt. Pune

Pune, India pharmaceutical TAC suspensions, differences in particle size amongst such products are predicted to be immaterial to their duration or performance.

KEY WORDS computational fluid dynamics (CFD) . convective diffusion - dissolution · drug clearance . intravitreal injection · volume of fluid (VOF)

\section{ABBREVIATIONS}

C drug concentration

$C_{a}$ mean aqueous humor concentration

$C_{v} \quad$ mean vitreous concentration

D drug diffusivity (region dependent)

E, F constants used in reference solution for single particle dissolution

$f \quad$ aqueous humor production rate.

$\mathrm{K}_{\mathrm{h}} / \mathrm{n}$ hydraulic conductivity (region dependent) divided by regional viscosity

$k_{v} \quad$ vitreous clearance rate

$R \quad$ radius of single drug particle for reference solution

$R_{0} \quad$ initial radius of single drug particle for reference solution

$R_{v} \quad$ radius of spherical vitreous region for reference solution

t time

TAC triamcinolone acetonide

$\rho \quad$ drug density

\section{INTRODUCTION}

TRIESENCE ${ }^{\mathrm{TM}}$ is a preservative-free suspension of $40 \mathrm{mg} / \mathrm{mL}$ Triamcinolone Acetonide (TAC) approved in the US for visualization during vitrectomy and for treatment of sympathetic ophthalmia, temporal arteritis, uveitis, and ocular inflammatory conditions unresponsive to topical corticosteroids. In the development of this product, a question 
arose regarding the potential impact of particle size on ocular bioavailability. The mean particle diameter measured for TRIESENCE $^{\mathrm{TM}}$ was 5-6 $\mu \mathrm{m}$. This was approximately onethird the particle size measured for KENALOG®-40, a suspension of TAC preserved with benzyl alcohol. Although KENALOG ${ }^{\circledR}-40$ was approved only for intramuscular and intraarticular use, it had been used off label to treat various ophthalmic conditions.

Assuming particles are spherically shaped, a three-fold decrease in particle diameter would require a 27-fold increase in the number of particles to maintain the same drug dose. The smaller particles would each have a surface area that is one-ninth that of the original particles, and there would be a three-fold increase in total particle surface area. The dissolution rate could be impacted by total particle surface area, and in fact, differences were observed in the in vitro dissolution rates measured for the two products. The method used was based upon USP Type II, which subjects the suspension particles to high rates of shear (stirring). But the hydrodynamic conditions to which suspension particles are exposed in the eye are vastly different. Before age 20, the vitreous is a quiescent gel. The central vitreous liquefies gradually upon aging (2). But even in the case in which a significant fraction of the vitreous has become liquefied, the rate of shear produced by eye movements is probably significantly lower than that of the USP dissolution test.

Thus, a series of simulations was conducted to determine the potential influence of particle size on dissolution rate of TAC suspension depots of varying designs placed in the vitreous of the rabbit eye. The geometric model was similar to other geometries used for in silico modeling of ocular drug disposition $(3,4)$, but was refined using insights afforded by high resolution NMR imaging (1). It will be demonstrated that the new geometric model provides superior predictions for the rate of clearance of various substances injected into the vitreous. This was accomplished by developing a computational model that is able to predict dissolution from the surface of individual particles. The validity of this method will be demonstrated by its ability to predict the dissolution rate of an individual particle in an idealized geometry. This computational model was then applied to ensembles of drug particles of various sizes. It will be demonstrated that the dissolution rate becomes independent of particle size once particle diameter decreases below a certain threshold value. In this limit, the dissolution rate matches the rates observed in vivo (5).

Prior to the simulation of particle dissolution, a separate study was conducted to simulate the clearance of fully dissolved material injected into the vitreous in three different geometric models for the rabbit ocular anatomy. One was the recent model advanced by Friedrich et al.(3), the second was a model of the whole rabbit eye based upon the geometry proposed by Park et al.(4), and the third is a new model proposed in this study. The three models were approximately equal in overall size and contained most of the same features, but differed in subtle yet important ways that affected the rate at which material is cleared anteriorly. The injected materials simulated were those for which clearance data was available, representing potential drugs spanning a range in molecular weight. The new geometric model proposed in this study showed the best agreement in predicting the available clearance data, and thus was selected for use in the particle dissolution simulations.

\section{MATERIALS AND METHODS}

\section{Software}

Computational Fluid Dynamics (CFD) calculations were conducted using FLUENT software version 6.3.26 (ANSYS, Inc., Canonsburg, PA). Geometrical models and meshing were constructed using GAMBIT version 2.4.16 (also developed and distributed by ANSYS). Simulations were conducted using a Dell Precision T5400 system with a Quad processor and 4 Gigs of RAM under 32-bit Windows XP. Because of memory limitations on this platform, some memory-intensive simulations required being run in parallel with a minimum of 2 processes.

\section{Geometry}

The geometry for the rabbit eye is shown in Fig. 1; key dimensions appear in Tables I and II. This model contains features that are similar to those used by others $(3,4,6,7)$ with the following unique distinguishing features. The shape of the posterior segment is oval, based upon the high resolution NMR imaging data in references $(1,8)$. The axial length and width of the eye and lens and the distances in Table I were estimated from Fig. 2 of reference 1 assuming the axial length in the figure was $1.6 \mathrm{~cm}$. Separate thin shell regions are defined for the retina and choroid. The structure of the anterior region (ciliary body, iris, and cornea) is based roughly on the model of Park (4), with dimensions guided by Heys $(6,7)$ and other information on ocular anatomy to guide the thicknesses and relative placement of the various tissues (9). The lens was not included in the model except for its shape, which was a void.

The model includes a $0.01 \mathrm{~cm}$ gap between the anterior boundary of the vitreous and the ciliary body, which effectively extends the posterior portion of the aqueous humor nearly to the ora serrata, the anteriormost portion of the retina. Anatomically, this has been designated as the retrozonular space of Petit, which was not represented in 

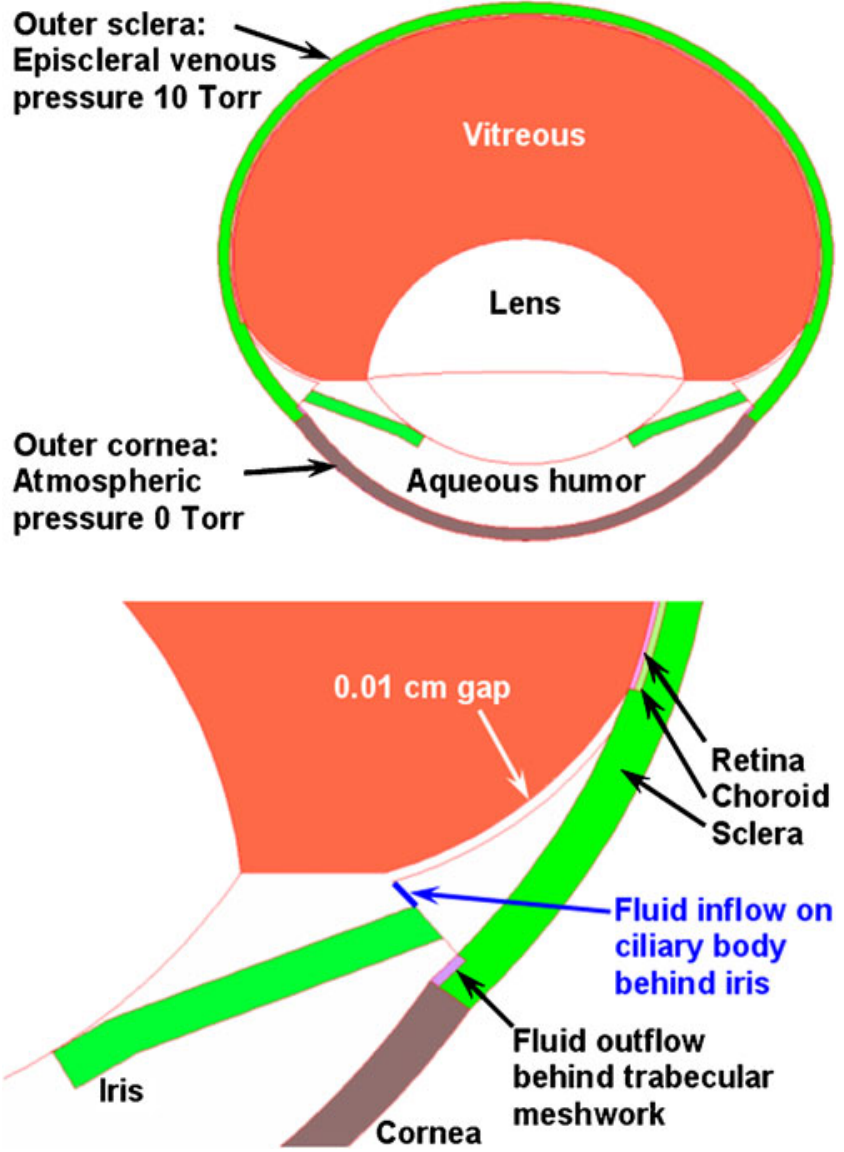

Fig. I Rabbit ocular geometry for the new model proposed in this study showing the various fluid regions and key surfaces for mass flow inlet and pressure outlet boundary conditions.

either the models of Friedrich et al. (3) or Park et al. (4). This gap has a significant physiologic impact facilitating clearance of certain intravitreally injected materials as it reduces the "bottleneck" effect restricting the efflux of materials that are not efficiently cleared through the outer vitreous (i.e., by the retina/choroid). Aqueous humor is produced on a circumferential band on the ciliary body behind the iris and is eliminated on a second band in front of the iris, at the forwardmost extent of the sclera, just outside the cornea, behind a ring of material representing the trabecular meshwork. The placement of the iris was similar to that of Heys et al. (6,7), allowing a distance of closest approach to the lens of 4 microns. To investigate the potential impact of various features of ocular geometry, similar whole-eye models were constructed for the Friedrich and Park geometries, as shown in Fig. 2. The volumes of the vitreous and aqueous compartments of each model are similar, but the ratio of the length to the width is quite different, mainly because of differences in the shape of the vitreous and the shape and size of the lens (Table III).

\section{Theory}

Three mechanisms for drug egress from the eye, in relative order of importance for small drug molecules such as TAC, are as follows:

- radial outward diffusion with subsequent clearance by the choroidal vasculature

- diffusion toward the front of the eye with clearance by the aqueous humor turnover

- hydraulic clearance by pressure-assisted convection through the outer sclera

Previous simulations of drug release from intravitreal devices indicated that intraocular pressure-driven convection would have minimal impact on drug delivery for small molecules $(10,11)$. The convection-diffusion equation accounts for both convection and diffusion of drug over time:

$\frac{d C}{d t}+\overrightarrow{\mathbf{v}} \cdot \nabla C=D \nabla^{2} C$

where $C$ is concentration, $D$ is the diffusion coefficient and $\overrightarrow{\mathbf{v}}$ is the velocity vector. The fluid velocity is obtained by solving for creeping flow in a porous medium. Using Darcy's Law, the local volume flux rate of fluid is related to the local pressure gradient by the equation (12):

$\overrightarrow{\mathbf{v}}=-\frac{K_{h}}{\eta} \nabla P$

where $P$ is the scalar pressure variable, $K_{h}$ is the permeability of the porous medium to fluid flow and $\eta$ is the fluid viscosity. Assuming conservation of matter, $\nabla \cdot \overrightarrow{\mathbf{v}}=0$ (13), we have that

$\nabla \cdot\left(\frac{\kappa_{h}}{\eta} \nabla P\right)=0$

Table I Key Dimensions of Structures for the Rabbit Eye (Dimensions in $\mathrm{cm}$ )

Axial length of eye 1.6

Width of eye at widest point

1.829

Distance, rear of eye to widest point

0.753

Distance, front of cornea to widest point

0.847

Distance, widest to flat portion of hyaloid

0.37

Distance, widest to lens rear

0.42

Distance widest to lens front

0.25

Width of lens at widest point

0.95

Thickness sclera

0.0328

Thickness choroid

0.0068

Thickness retina 0.0051 
Table II Key Radii and Coordinates for the Rabbit Eye Model (Dimensions in $\mathrm{cm}$ )

\begin{tabular}{lllll}
\hline & RI & R2 & X-center & Z-center \\
\hline Outer sclera & 0.9142857 & 0.7525275 & 0 & 0 \\
Choroid-sclera boundary & 0.8814857 & 0.7197275 & 0 & 0 \\
Retina-choroid boundary & 0.8746857 & 0.7129275 & 0 & 0 \\
Vitreous-retina boundary & 0.8695857 & 0.7078275 & 0 & 0 \\
Lens rear & 0.478601 I & 0.478601 I & 0 & -0.428601 I \\
Lens front & 0.5762498 & 0.5762498 & 0 & -0.0437502 \\
Cornea outside & 0.8479267 & 0.8479267 & 0 & 0.0004540 \\
Cornea inside & 0.7898060 & 0.7898060 & 0 & -0.0216670 \\
Curved portion of hyaloid & 0.3836340 & 0.3836340 & 0.5067752 & 0 \\
Curved cb adjacent to hyaloid & 0.3936340 & 0.3936340 & 0.5067752 & 0 \\
Intersection lens equator with hyaloid & & & 0.475 & -0.37 \\
Intersection curved \& flat hyaloid & & & 0.608141 & -0.37 \\
Intersection curved hyaloid \& retina & & & 0.834151 & -0.2 \\
Intersection outer sclera, outer cornea & & & 0.678 & -0.496548 \\
Intersection inner sclera, inner cornea & & & 0.650239 & -0.469978 \\
Intersection cb, tm, aqueous, sclera & & & 0.675495 & -0.44575 I \\
\hline
\end{tabular}

Eqs. 1-3 are written in general form for differential operators, and would take on more specific forms in selected geometries.

\section{Material Properties, Initial Conditions, Boundary Conditions}

The aqueous humor is a simple fluid region with the viscosity of water. All remaining regions are porous media (porosity $=1$ ) with the hydraulic resistance values assigned in Table IV. The band on the ciliary body behind the iris, in direct contact with the aqueous humor, was a mass flow inlet. The aqueous humor production rate used on this inlet was set to $3 \mu \mathrm{L} / \mathrm{min}\left(2.5 \times 10^{-8} \mathrm{~kg} / \mathrm{s}\right.$ for the hemisphere), based upon the measurements of Reitsamer $(14,15)$. This is

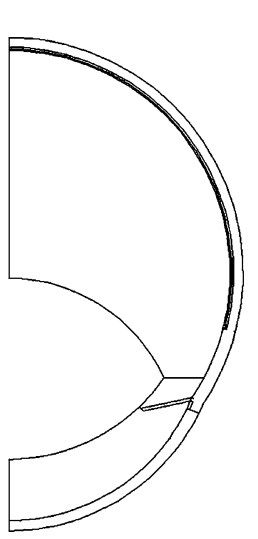

Friedrich

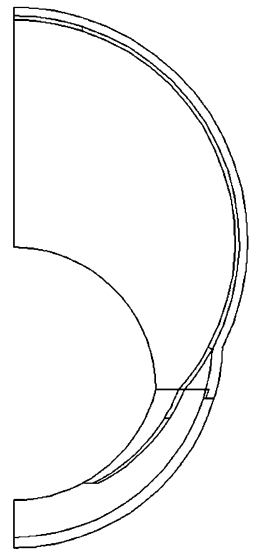

Park

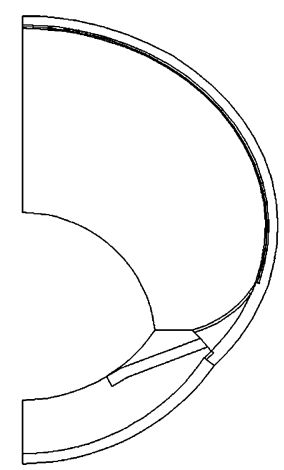

New
Fig. 2 Cross-sectional view of the ocular rabbit eye geometries for the three different models examined. The geometry labeled "New" pertains to the model proposed in this study. moderately higher than the value of $2.2 \mu \mathrm{L} / \mathrm{min}$ used by Friedrich (3) and Park (4), which were apparently based upon the earlier measurements of Kinsey (16). The band behind the trabecular meshwork was a pressure outlet with a zero gauge pressure. The outer sclera was maintained at a gauge pressure of 10 Torr, representing the episcleral venous pressure (17-19). The resistance for the vitreous was taken from the literature (20). The last value in Table IV, for other tissue regions (not including aqueous humor), was taken from measurements of scleral resistance (21). The value for the trabecular meshwork was adjusted to provide a maximum intraocular pressure of 15 Torr. A slightly different value was required to achieve this pressure difference in the various geometries (see Table IV).

Two types of problems were run, requiring two separate initial conditions. The first type was a simple bolus injection of a diffusing material (e.g. drug) entirely in the dissolved state. The initial condition was modeled by localizing all drug in a $10 \mu \mathrm{L}$ bolus in the central vitreous close to the rear of the lens. This was modeled as a convection-diffusion problem, in which the flow solution was obtained first, solving only for the pressure and velocity variables in the domain. The drug distribution was obtained by solving a transient user-defined scalar equation in the steady-state

Table III Characteristics of the Various Ocular Models

\begin{tabular}{llll}
\hline & Friedrich & Park & New \\
\hline Vitreous volume $(\mathrm{mLs})$ & 1.462 & 1.579 & 1.7 \\
Aqueous volume $(\mathrm{mLs})$ & 0.234 & 0.245 & 0.252 \\
Axial length $(\mathrm{mm})$ & 17.33 & 19.02 & 16.00 \\
Width $(\mathrm{mm})$ & 8.25 & 8.25 & 9.14 \\
\hline
\end{tabular}


Table IV Hydraulic Resistance Values Assigned to Various Tissue Regions

\begin{tabular}{ll}
\hline Tissue & $\begin{array}{l}\text { Hydraulic Resistivity } \\
\left(\mathrm{m}^{-2}\right)\end{array}$ \\
\hline Vitreous & $1.19 \times 10^{13}$ \\
Trabecular Meshwork, Park eye model & $2.84 \times 10^{15}$ \\
Trabecular Meshwork, Friedrich rabbit model & $2.51 \times 10^{15}$ \\
Trabecular Meshwork, new rabbit model & $6.36 \times 10^{15}$ \\
Interior of undissolved drug bead & $1.19 \times 10^{21}$ \\
All other tissues except aqueous humor & $6.67 \times 10^{17}$ \\
\hline
\end{tabular}

fluid velocity field. This approach was used to compare the clearance rates of diffusing materials in the various rabbit model geometries. The time course over which the advection was simulated was $5 \times 10^{5} \mathrm{~s}$ for small molecules (sucrose/fluorescein glucuronide and fluorescein) and $1.2 \times$ $10^{6} \mathrm{~s}$ for all other materials, which diffused more slowly. The time increment was reduced until results became independent of the timestep. This occurred for $500 \mathrm{~s}$ timesteps in the case of fluorescein and sucrose and 5,000 s timesteps for all other materials.

The second type of problem modeled the dissolution of TAC drug crystals directly. Therefore, TAC was present in both solid and dissolved states. These two states were defined as separate and distinct phases in the model. At time $=0$, all drug was in the solid (undissolved) phase. This was accomplished by patch-initializing the user-defined scalar to a constant value in the region(s) where drug exists initially, either in a single spherical pellet or distributed amongst suspension particles arranged geometrically in the eye according to a particular design. Thus, the potential impact of dissolved drug in the injected bolus (comprising up to $96 \%$ of the volume but containing an insignificant fraction of drug compared to that in the suspension crystals) was ignored. The particles and vitreous gel were treated as immiscible phases in our model; therefore, FLUENT's Volume of Fluid (VOF) multiphase method was used to simulate dissolution from the surface of the drug crystal(s). A user-defined function (UDF) was programmed to adjust the location of the drug-gel interface based upon the flux of drug species from the surface at a given point. The drug is set to the solubility limit of $36 \mathrm{ppm}$ at the dissolving interface (22). At each timestep, the system measures the amount of drug transported away from the dissolving surface by convection and diffusion, and the UDF moves the boundary in such a manner so as to reduce the local volume of the drug particle by an appropriate quantity to offset the mass of drug transported within the simulated time increment. The rate of transport from each point on the surface of a given particle is determined by the local concentration gradient and by the magnitude of the fluid velocity in that region. Hence, drug dissolution rates will vary regionally for different particles at various locations within the vitreous and also for different regions of an individual particle, depending on the local environment. The eye geometry of Fig. 1 was used for this investigation.

Particle dissolution simulations were conducted solving all variables simultaneously: pressure, the three components for fluid velocity, the two phases, and drug concentration throughout the dissolved phase. The initial time increment was extremely small $(1 \mathrm{~s})$, and the time increment was kept small during the initial establishment of the quasi-steadystate distribution of dissolved drug concentration. Once this drug distribution was established, the time increment could be increased to $50,000 \mathrm{~s}$, which represented $0.25-1 \%$ of the time to total suspension dissolution, providing adequate discretization of the dissolution process. It was verified that the simulated particle dissolution rate was not affected by the timestep size. Simulations could be conducted to complete particle dissolution in 50-100 CPU hours depending upon the complexity of the suspension design.

The values of drug diffusivity in the vitreous used for various materials appear in Table $\mathrm{V}$. The value for TAC and sucrose (or fluorescein glucuronide) was the same as the value typically used by Maurice and co-workers for fluorescein, a molecule of comparable molecular weight (see for example Kaiser and Maurice (23)). The value for albumin was taken from Maurice (24). The values for the Dextran polymers were the same as used in Missel (11). The value for $67 \mathrm{kD}$ was assumed to be $6 \times 10^{-11} \mathrm{M}^{2} \mathrm{~s}^{-1}$ (Araie and Maurice (25), Maurice (26)), and the values for the other Dextran polymers were calculated assuming the diffusivity scales with the cube root of the molecular mass (27). Diffusivity in the aqueous humor was set to that in vitreous.

For the bolus injection studies, the value of diffusivity in the sclera and cornea was the value in Table $\mathrm{V}$ divided by 13.33, and the value of diffusivity in the retina and choroid was one-tenth the value in the sclera/cornea. These ratios were identified from studies on the transport of anecortave acetate in the vitreous and in posterior ocular tissues (28). The ratio between the diffusivities in the vitreous and sclera was roughly comparable to estimates of other workers, who

Table V Values of Vitreous Diffusivity Used for Various Materials

\begin{tabular}{ll}
\hline Material & $\begin{array}{l}\text { Diffusivity } \\
\left(10^{-10} \mathrm{~m}^{2} \mathrm{~s}^{-1}\right)\end{array}$ \\
\hline Triamcinolone Acetonide & 6.0 \\
Fluorescein, Fluorescein Glucoronide, Sucrose & 6.0 \\
Albumin & 0.84 \\
I0.5 kD Dextran & 1.1 \\
$67 \mathrm{kD}$ Dextran & 0.6 \\
I57 kD Dextran & 0.45 \\
\hline
\end{tabular}


found this ratio to be 1:6 for the transport of small (29) and large (30) molecules through the sclera. A low value for diffusivity in the peripheral tissues effectively shuts them off from being significant routes for drug elimination. Since the main focus of the current studies is clearance from the vitreous, this crude one-size-fits-all estimate for diffusivity ratios in various tissues is sufficient. Predictions of drug concentrations in the peripheral tissues themselves would require a more accurate treatment of diffusivity in retina, choroid and sclera. The particular value of diffusivity in the iris, ciliary body and trabecular meshwork, set equal to the value in the choroid and retina in these studies, would have little impact on the clearance from the vitreous.

The tissue diffusivities assigned for the TAC dissolution studies were as follows. The diffusivity in vitreous, and aqueous humor was set to $6 \times 10^{-10} \mathrm{~m}^{2} \mathrm{~s}^{-1}$. The value in all other tissues was set to $4.5 \times 10^{-12} \mathrm{~m}^{2} \mathrm{~s}^{-1}$ (133.3 times lower). This strategy was slightly different from that of the bolus injection studies in two ways. First, there was no distinction in the diffusivity in the sclera, cornea, choroid, iris or ciliary body. Second, the diffusivity in the retina matched that in the vitreous in order to maximize the sink effect at the exterior vitreous (see next paragraph). Since the egress of drug from the system was mainly by means other than transport through peripheral tissues, the rate of drug elimination in either type of study was not impacted by the particular value of diffusivity in peripheral tissues.

Boundary conditions on concentration were as follows. The value of the user-defined scalar was set to zero on the fluid inlet at the ciliary body. For the bolus injection simulations, this was the only boundary condition for the diffusant, except in the case of fluorescein, in which an infinite sink was applied in the retina, iris, and ciliary body, to reflect the activity of an active transport mechanism effective in these tissues. For all other materials the predominant means of egress for drug, apart from the insignificant transport through peripheral tissues, was by diffusion into the posterior chamber, admixing with aqueous humor and elimination through the trabecular meshwork. For the intravitreal TAC suspension simulations, an infinite sink was applied in the retina only.

Three types of TAC suspension models were designed: $4 \mu \mathrm{L}$ drug confined to a $100 \mu \mathrm{L}$ spherical depot, $16 \mu \mathrm{L}$ drug confined to a $100 \mu \mathrm{L}$ spherical depot, and $4 \mathrm{mg}$ drug dispersed uniformly throughout the entire vitreous. Suspensions of each of these types were comprised of equisized spherical particles of varying particle size. Particle locations were calculated based upon the hexagonally close-packed $\alpha$ La (A3') crystal lattice structure (http://cst-www.nrl.navy.mil/lattice/struk/ a3p.html). The interparticle spacing was fine-tuned to be made just large enough so as to include the specified number of particles within the region without excluding any additional particles, accounting for the diameter of the particle calculated. In this manner, the suspension for a particular number of particles would be expanded just to the point in which all of the particles would be contained within the volume, with some of the particles on the outside just touching the outer boundary of the depot.

Particles were not constructed explicitly in the original mesh. Rather, the mesh adaption facility in FLUENT was used to refine the mesh near the particle regions from an existing mesh of half the rabbit eye geometry. This required that the mesh after adaption be sufficiently refined in the region of the depot such that each particle be comprised of at least 30 cells upon marking the particle regions. For suspensions of smaller particles, it was necessary to further refine the particles by either one or two adaption cycles. For the case in which the suspension was dispersed throughout the entire vitreous, the geometry was reduced to a wedge representing one-twelfth of the eye. This enabled sufficient mesh refinement of the entire vitreous region without exceeding memory limitations. The mesh was refined as necessary to provide an initial calculated total particle volume that was within $1 \%$ of the target.

In the Appendix, an exact analytical mathematical relationship is derived for the time dependence of dissolution by surface erosion of a spherical drug bead suspended in the center of a vitreous shell surrounded by an infinite sink (approximating the choroidal clearance). The simulation method was validated by verifying that it can accurately predict the dissolution time course for this idealized situation. The initial condition was a $4 \mu \mathrm{L}$ spherical bead placed in the center of a $4 \mathrm{~mL}$ spherical vitreous. No pressure or flow was involved. Vitreous drug diffusivity and all other properties were identical to those used for the particle dissolution simulations.

\section{RESULTS}

The orderly flow pattern in the aqueous humor is visualized by the pathlines of flow, seeded from the fluid inlet, in Fig. 3. Fig. 4 a presents a contour plot of pressure. The entire vitreous and aqueous humor compartments are at or very close to the assigned intraocular pressure (15 Torr), with the pressure drop occurring almost entirely across the sclera and cornea. Fig. 4b presents a contour plot of fluid velocity magnitude, scaled to a maximum velocity of $10^{-8} \mathrm{~m} / \mathrm{s}$ to illustrate the velocity distribution in the vitreous. Fig. 4c illustrates a contour plot of the steady-state TAC concentration for a representative spherical suspension depot early in the dissolution process, after the drug concentration has achieved a quasi-steady-state, but before a significant amount of material has dissolved. Also plotted in the figure are the particle surfaces.

To illustrate the influence of the different modes of elimination for these two materials, Fig. 5 shows represen- 


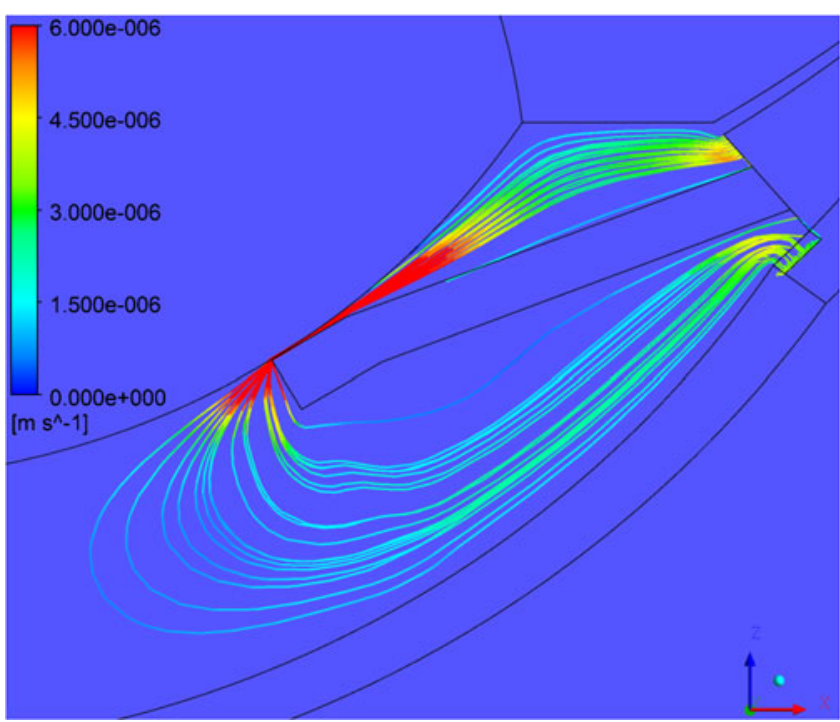

Fig. 3 Pathlines of flow seeded from the fluid inlet to the posterior aqueous humor. Colors indicate velocity $(\mathrm{m} / \mathrm{s})$. The velocity displayed is clipped to a maximum value of $5 \mathrm{E}-6 \mathrm{~m} / \mathrm{s}$.

tative concentration contours for fluorescein (Fig. 5a) and $157 \mathrm{kD}$ Dextran (Fig. 5b) at different times after bolus injection. Fig. 6 compares the clearance rates of the various materials as measured experimentally $(26,27,31-33)$ together with clearance rates estimated from simulations for the three models of Fig. 2. Plotted in Fig. 6 are results for the ratio of the mean aqueous and vitreous concentrations versus the clearance rate. The slanted line represents a prediction for materials that are eliminated only by the anterior pathway (see Eq. 4, discussed below). The influence of varying intraocular pressure for the geometric model of Fig. 1 is shown in Fig. 7, over the range of 10.1-20 Torr. These pressures were obtained by reducing the hydraulic resistance of the trabecular meshwork to a negligible value to achieve a pressure barely above the episcleral venous pressure of 10 Torr, to approximately twice the setting for the normotensive condition to achieve the higher pressure.

Validation of the multiphase dissolution model outlined in the Theory section is shown in Fig. 8 for a spherical particle suspended in an idealized spherical vitreous domain. The model was capable of simulating surface dissolution by tracking the movement of the dissolving front through an existing mesh. The two graphical insets in Fig. 8a illustrate the shape of the drug bead at initial time and for a time after $50 \%$ of the drug has dissolved for the spherically symmetric reference problem. The dissolution versus time profile predicted by the analytical result of the Appendix was reproduced with great accuracy. The significance of the other mathematical dependencies compared is discussed below. Curves for the time dependence of the dissolution rate for the various models (the magnitude of the slopes of the curves) appear in Fig. 8b.
The validated framework was then applied to the dissolution of particle suspensions. Fig. 9 illustrates selected particle arrangements that were simulated with the VOF model. A quantitative measure of the status of the dissolution process as a function of time is provided by Fig. 10, which plots the total undissolved drug for the case of $4 \mathrm{mg}$ drug divided amongst equally sized particles of
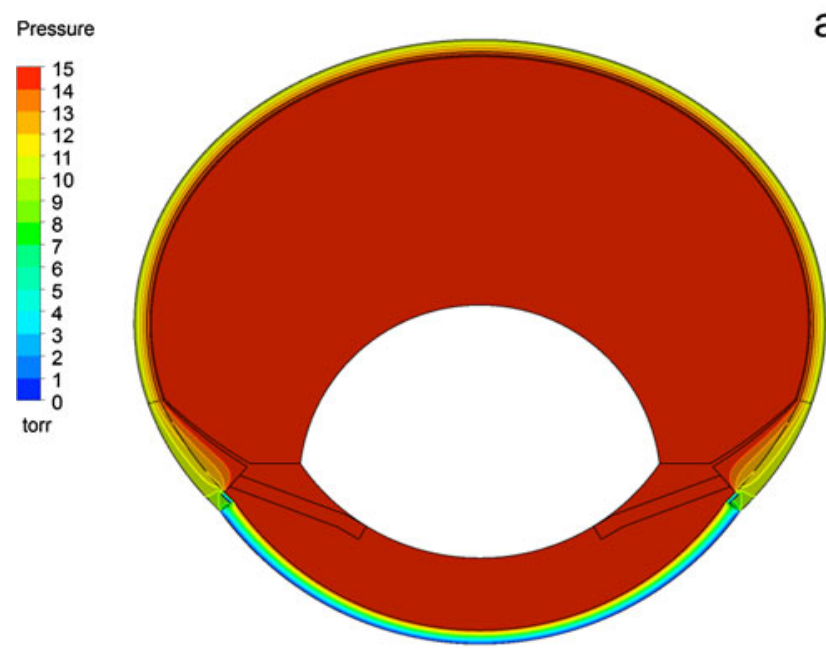

a)

b)
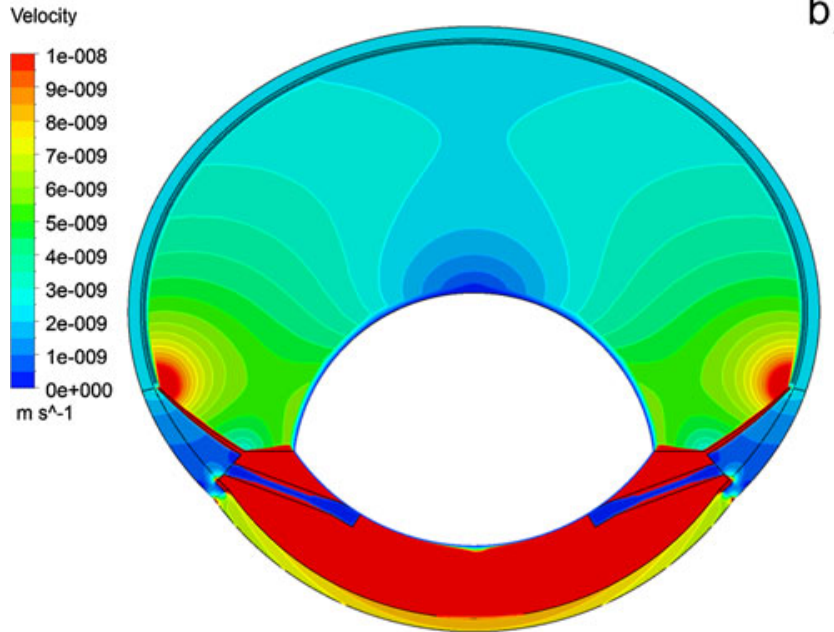

c)

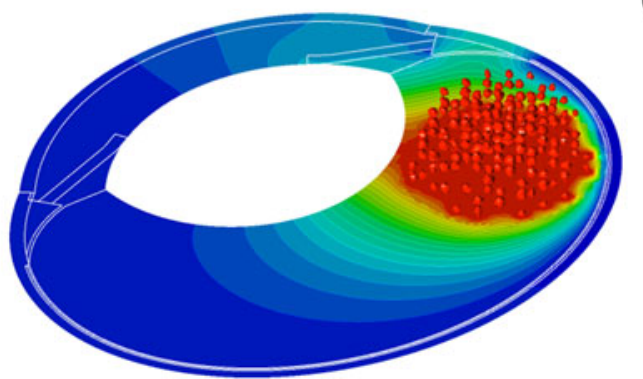

Fig. 4 a Pressure displayed on the symmetry surfaces (Torr). b Velocity, clipped to a maximum value of $10^{-8} \mathrm{~m} / \mathrm{s}$ to illustrate contours in the vitreous. c Dissolved Triamcinolone Acetonide concentration (grams/ml) after the dissolution process has achieved steady state for a suspension of $16 \mathrm{mg}$ Triamcinolone Acetonide divided into 276 particles confined to a 100 microliter intravitreal bolus. 


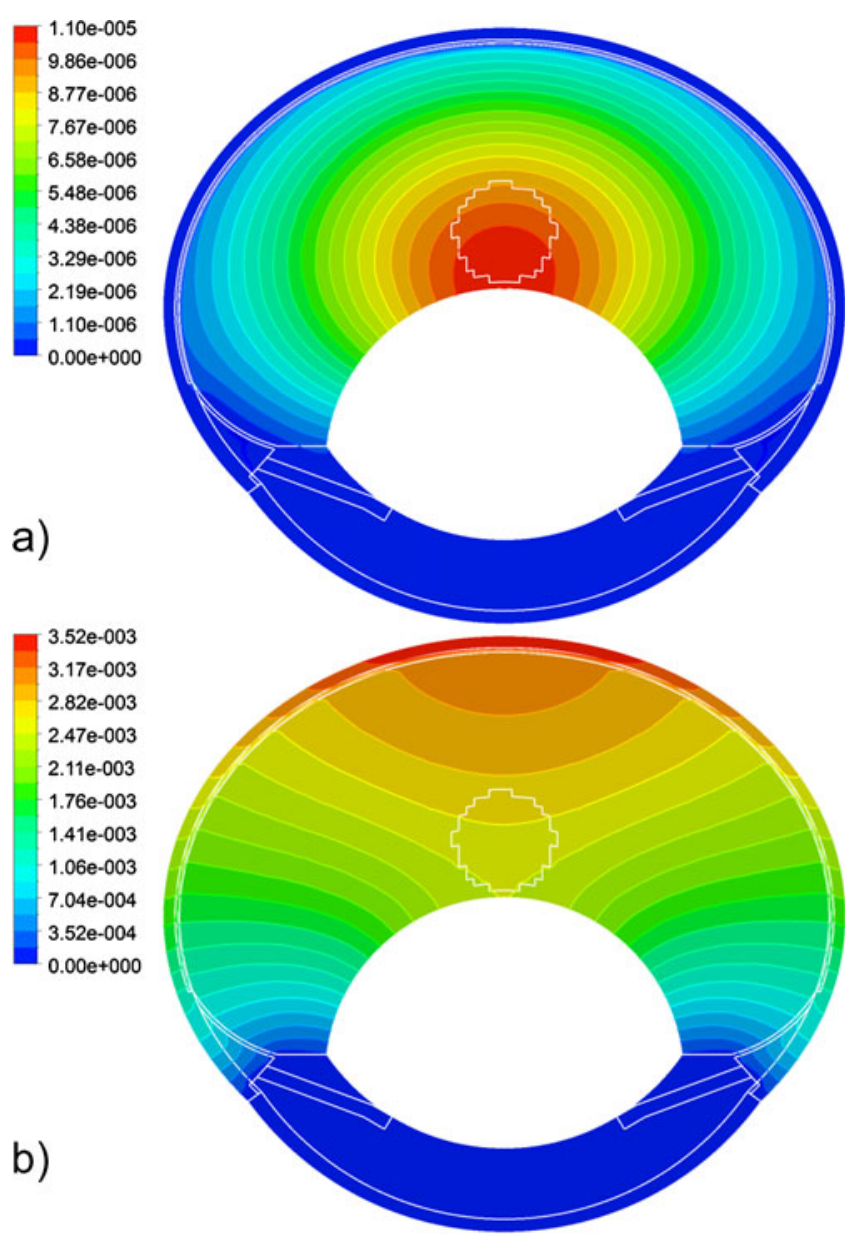

Fig. 5 Concentration profiles following central bolus injection of $10 \mu \mathrm{L}$ intravitreal injection of a substance of concentration I (arbitrary units) in the current rabbit model. a Fluorescein, $10^{5} \mathrm{~s}$ after injection. b $157 \mathrm{kD}$ Dextran, $10^{6} \mathrm{~s}$ after injection.

various sizes confined to a $100 \mu \mathrm{L}$ spherical depot. Data points are from Kim et al. (5). A general feature of the release profiles for the various studies is that the dissolution rate increases and the duration to final dissolution decreases systematically as the material is divided into smaller particles. This trend continues until a point is reached at which the profile appears to become independent of particle size, as exhibited by the convergence of the curves in Fig. 10. A convenient way to compare the influence of particle size on dissolution rate is shown in Fig. 11, in which the duration (time to total disappearance of undissolved drug) is plotted versus the area ratio, defined as the total initial suspension particle surface area normalized to that for the largest particles modeled. When plotting the duration data in this manner, it was discovered that the same very simple kinetic equation provided an excellent fit for all of these studies: $\mathrm{y}=\mathrm{a}+\mathrm{b} \exp (-\mathrm{ct})$. The particle size for each configuration below which further reduces in size will not reduce the suspension lifetime by more than $1 \%$ are indicated on each of the plots and are as follows. For a suspension in which $4 \mathrm{mg}$ drug is dispersed throughout the entire vitreous, the size-independent limit is obtained at a particle diameter of $77 \mu \mathrm{m}$. When this same $4 \mathrm{mg}$ of drug is confined to a $100 \mu \mathrm{L}$ spherical depot within the vitreous, the particle diameter at which the size-independent limit is reached increases to $167 \mu \mathrm{m}$. If the amount of drug within this $100 \mu \mathrm{L}$ spherical depot is increased from $4 \mathrm{mg}$ to $16 \mathrm{mg}$, the particle diameter at which the size-independent limit is reached increases further to $428 \mu \mathrm{m}$.

Fig. 12 compares the size-independent results for the 4 and $16 \mathrm{mg}$ spherical depots with the experimental data of Kim et al. (5). The predicted profiles for the aqueous humor concentration appear in the inset. The simulation for the $16 \mathrm{mg}$ depot lasts about 8 months, similar to the experimental data. The simulation for the $4 \mathrm{mg}$ depot last about 2 months, somewhat shorter than the nearly 3 -month duration observed in vivo in both man and rabbit in various studies $(5,34)$. The simulations assumed the volume of the depot remains constant at $100 \mu \mathrm{L}$, and the particle positions remain stationary, whereas Kim et al. observed that the depots tended to condense with time (5). To crudely simulate this effect, the simulation was halted after 10 days, at which point the remaining mass of $2.7 \mathrm{mg}$ was redistributed amongst 293 particles, each particle $260 \mu \mathrm{m}$ in diameter, confined to a smaller $25 \mu \mathrm{L}$ spherical depot. The results of this simulation were delayed 10 days and plotted as the dashed curve in Fig. 12.

Fig. 13 compares the aqueous humor concentration versus time profiles for suspensions comprised of 19 equal-sized particles confined to a $100 \mu \mathrm{L}$ spherical depot in three different locations within the vitreous, spanning the extreme rearward and forward locations available. The duration to total dissolution is virtually identical for all three locations.

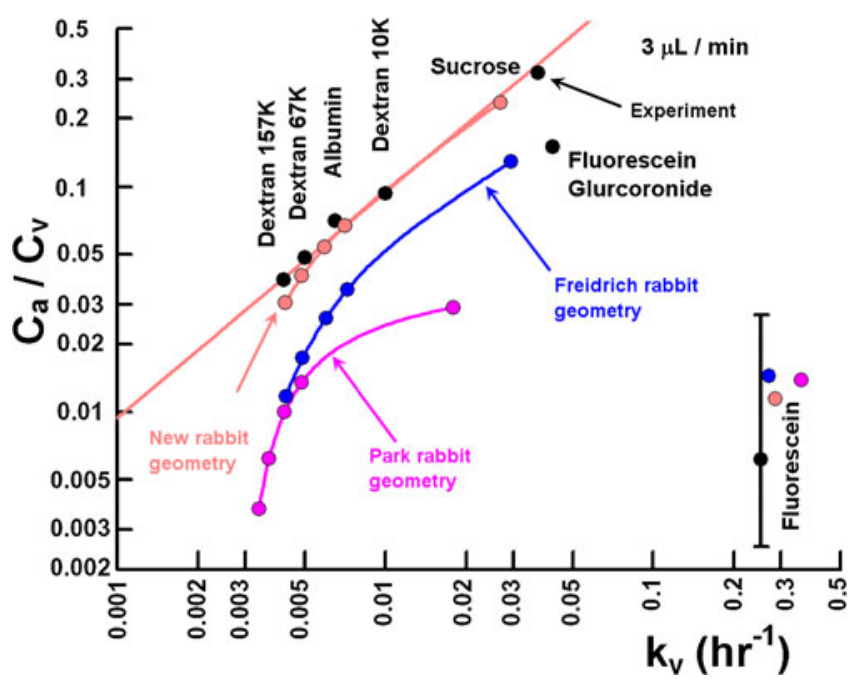

Fig. 6 The clearance rates of various materials injected into the rabbit vitreous as predicted by the three ocular models compared with experiment. 


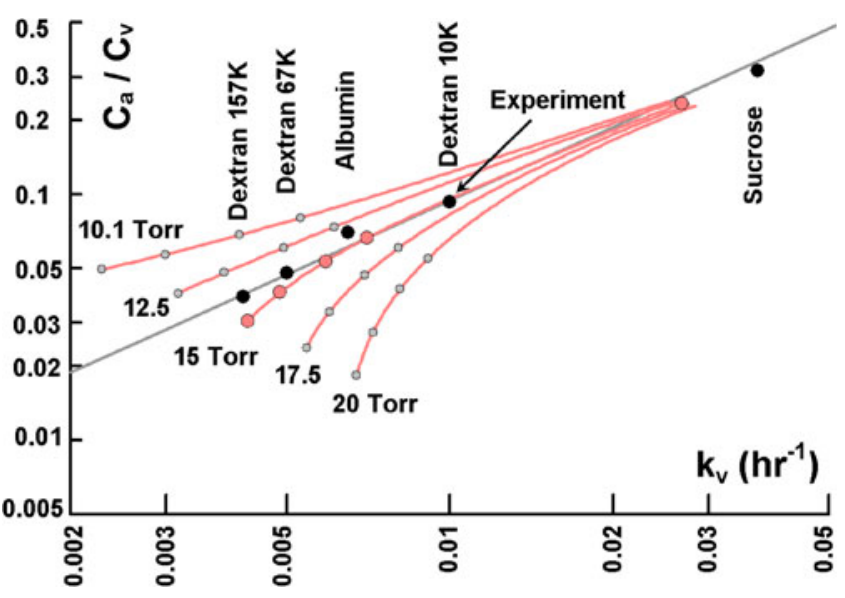

Fig. 7 Influence of intraocular pressure on the clearance rate of materials injected into the rabbit vitreous for the current geometry. The pressures indicated represent the maximum pressure in the eye.

The aqueous humor concentration profiles show quite similar shapes and exhibit higher maximum concentrations the more forward the depot is placed within the vitreous. The fraction of drug flux exiting by the anterior route at an early dissolution time of 5E5 seconds was calculated to be $8.7,5.0$, and $3.9 \%$ of the total drug flux out of the suspension depot for locations $\mathrm{A}, \mathrm{B}$, and $\mathrm{C}$ as shown in the inset.

\section{DISCUSSION}

One key assumption that is typically made by most models of this type is that material diffusivity of small molecules in the vitreous is identical to that in aqueous humor. This equivalence has been supported by recent experimental evidence indicating that the medium in which diffusion occurs for small molecules in the vitreous gel is the aqueous phase (35). This approach will not hold for very large molecules or for microparticles, as eventually the vitreous gel will exhibit diffusive resistance and slow the diffusivity compared to that in pure water (36). By the time the diffusing substance increases in size such that it becomes a particle of a few microns, the diffusivity is slow enough that the particles are essentially locked into the vitreous gel. The diffusivities of dissolved drug in the peripheral tissues are quite slow, and one may be tempted to discount transport in these outer regions. If this is done, the clearance out of the vitreous might be estimated accurately enough, but this approach would not allow for the prediction of diffusing material concentration in the peripheral tissues. Predictions of delivery to these tissues would be possible to the extent the diffusion coefficients were available, perhaps by independent transport measurements (37).

The velocity distribution in Fig. 4b is quite comparable to the result of the Park model (4) for the normotensive rabbit eye. Note that in their model, flows resulted from mass flow conditions placed on the ciliary body $(2.2 \mu \mathrm{L} / \mathrm{min})$ and the outer vitreous $(0.1 \mu \mathrm{L} / \mathrm{min}$ for the normotensive eye, based upon an in vivo estimate (25) of $0.097 \mu \mathrm{L} / \mathrm{min}$ ), with the balance of fluid cleared through the equivalent of the trabecular meshwork, and pressures resulted from these imposed flows. In the current model, the only mass flow boundary condition was for fluid production at the ciliary body ( $3 \mu \mathrm{L} / \mathrm{min}$ ), and the hydraulic resistance of the trabecular meshwork was manipulated to produce the desired intraocular pressure. The fluid efflux rates which result in the current model through the outer vitreous and outer sclera are 0.075 and $0.089 \mu \mathrm{L} / \mathrm{min}$, respectively, quite comparable with the estimate of Araie and Maurice (25).

The contours in the two plots of Fig. 5 are quite different because of the different ways in which drug is eliminated from the eye for these two materials. The contours resemble those observed in the study by Araie and Maurice (25), and those calculated previously using the Friedrich model for the rabbit posterior segment (38). Materials
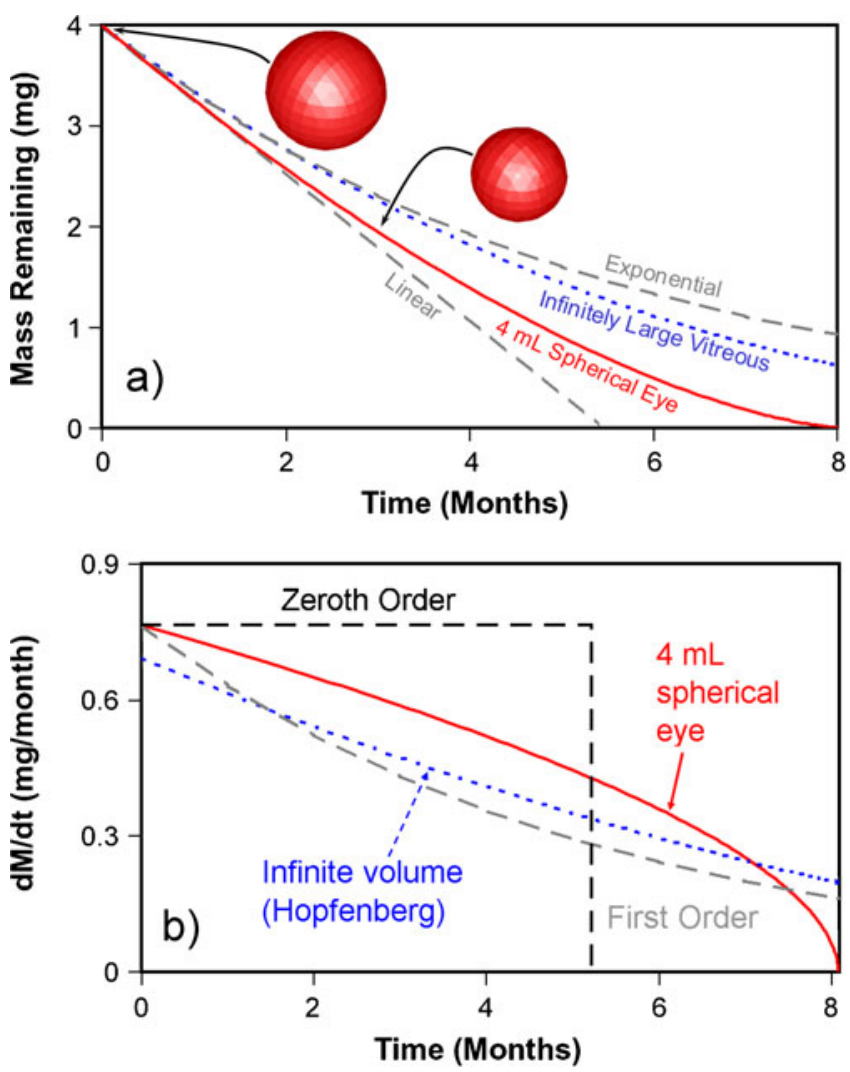

Fig. 8 a Validation of the method for simulating surface erosion of a $4 \mathrm{mg}$ spherical drug particle assuming a solubility limit of $36 \mathrm{ppm}$, centered in a $4 \mathrm{~mL}$ spherical vitreous surrounded by an infinite sink. The inset shows the initial particle shape and the shape after half the material has eroded, demonstrating the orderly advancement of the dissolving front. The numerical results are indistinguishable from the exact solution (solid curve). The prediction for an infinitely large vitreous expanse (2) is included for reference (dotted curve). Also shown for comparison are linear and exponential curves extrapolated from the initial dissolution rate (dashed curves). $\mathbf{b}$ Rate of mass loss for the same model. 


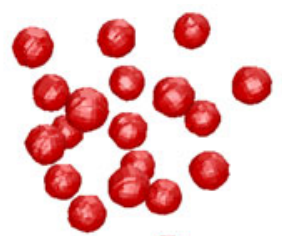

a)

d)

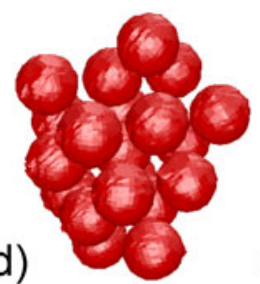

b)

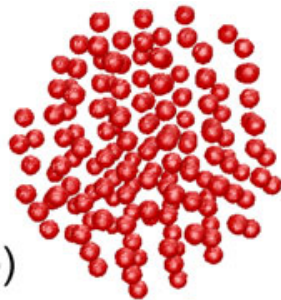

e)

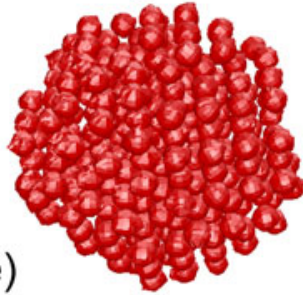

c)
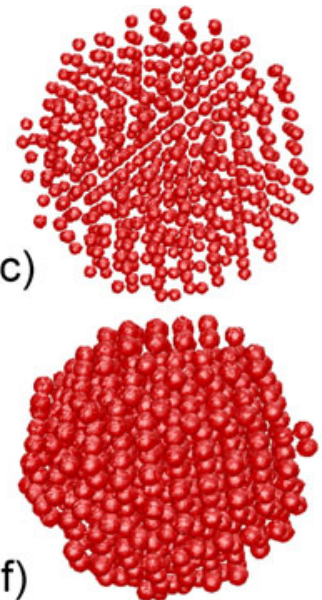

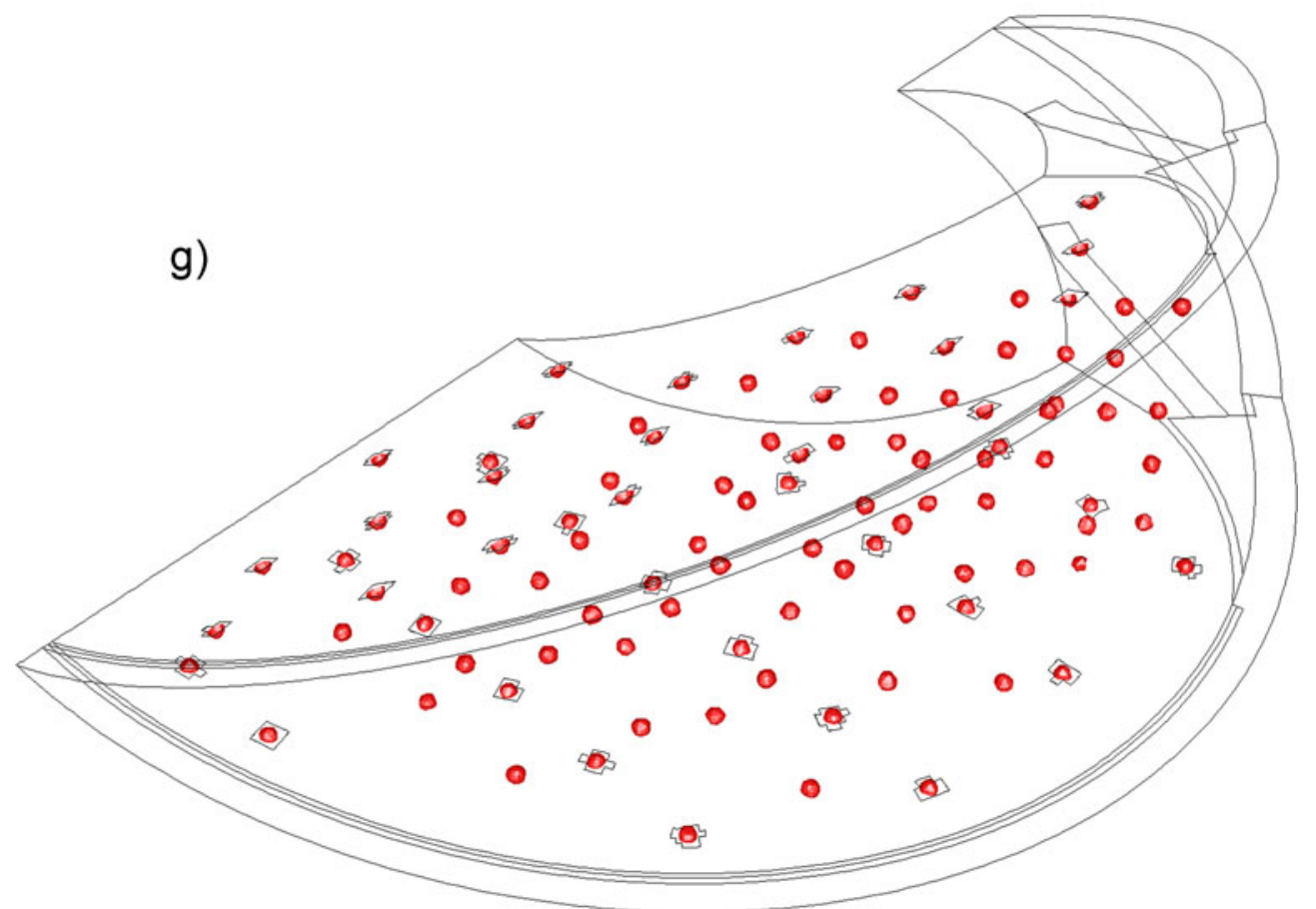

Fig. 9 Arrangements for particle suspensions. $4 \mathrm{mg}$ suspensions confined to I00 $\mu \mathrm{L}$ spherical depot: a 19 particles (738 $\mu \mathrm{m}) ; \mathbf{b}$ I47 particles (373 $\mu \mathrm{m})$; c 5 I 5 particles $(246 \mu \mathrm{m})$. $16 \mathrm{mg}$ suspensions confined to $100 \mu \mathrm{L}$ spherical depot: d 21 particles (I I33 $\mu \mathrm{m})$; e I59 particles $(577 \mu \mathrm{m}) ; \mathbf{f} 588$ particles $(373 \mu \mathrm{m}) . \mathbf{g} 4 \mathrm{mg}$ dispersed uniformly throughout the entire vitreous, I/I 6th of the model shown. When replicated the entire vitreous contains 942 particles $(201 \mu \mathrm{m})$. Sizes quoted are particle diameters.

which are cleared efficiently by transport through the outer vitreous (such as fluorescein) will have concentration contours resembling those of Fig. 5a, whereas materials that are cleared entirely by the anterior pathway (such as Dextran) will have contours resembling those of Fig. 5b.

The slanted line in Fig. 6 was calculated using a simple mathematical relationship derived for materials that are only eliminated by the anterior pathway, as follows (27):

$\frac{C_{a}}{C_{v}}=\frac{k_{v} V_{v}}{f}$ where $C_{a}$ is the mean aqueous concentration, $C_{v}$ is the mean vitreous concentration, $k_{v}$ is the vitreous clearance rate, and $f$ is the aqueous humor production rate. A vitreous volume of $1.7 \mathrm{~mL}$ was assumed, from the new ocular geometry, and a value of $3 \mu \mathrm{L} / \mathrm{min}$ for $f$. The experimental points for hydrophilic materials not cleared by the choroid come close to this line and demonstrate the same slope over a broad range in elimination rate. This plot provides a sensitive way to examine how well the simulations reproduce clearance behavior for materials cleared only by the anterior pathway. The point for fluorescein falls quite far away 


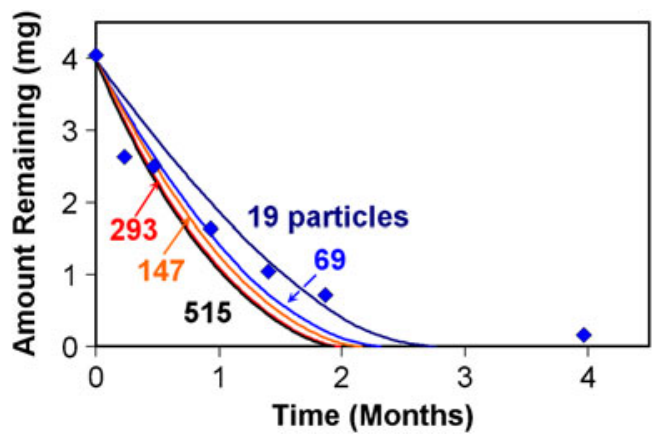

Fig. I0 Influence of the number of particles on dissolution for suspension models comprised of $4 \mathrm{mg}$ of equally sized particles confined to a $100 \mu \mathrm{L}$ spherical depot. Data points are from reference 5.

from the curve, because of the active transport mechanisms operational in the retina and in the anterior tissues. The infinite sink in the simulations applied in the retina is mainly responsible for increasing the value of $\mathrm{k}_{\mathrm{v}}$, whereas
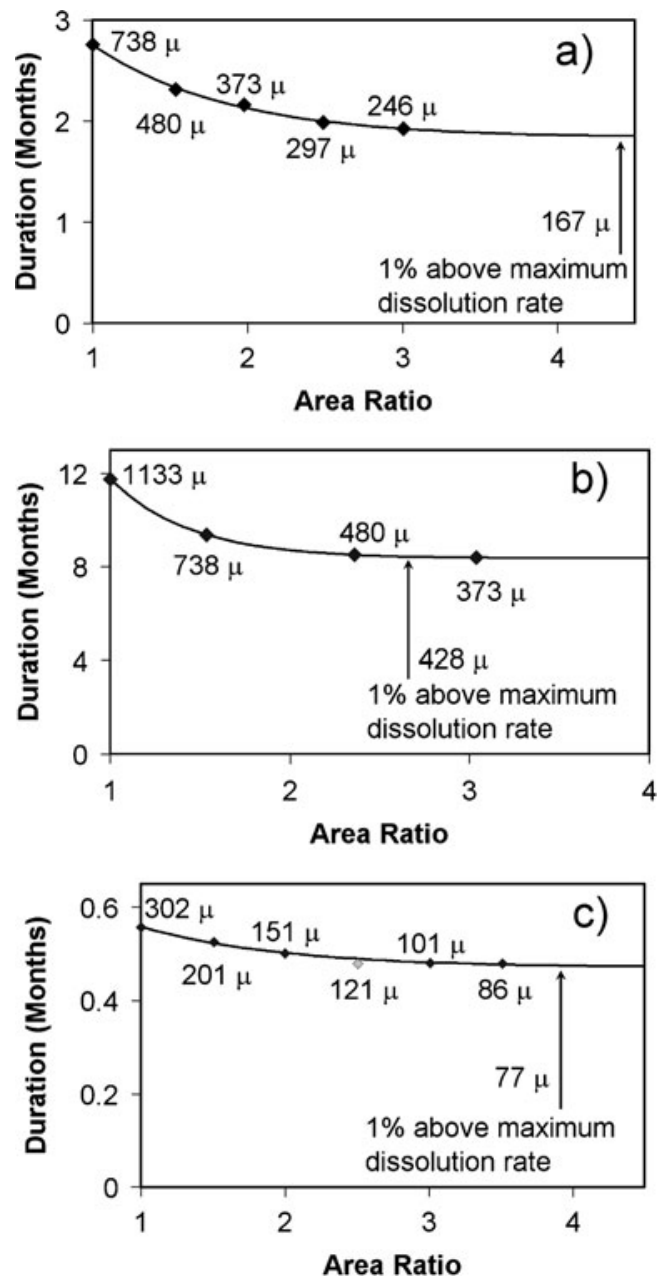

Fig. I I Influence of particle size on the duration of intravitreal suspensions. a $4 \mathrm{mg}$ TAC confined to a $100 \mu \mathrm{L}$ spherical depot. b $16 \mathrm{mg}$ TAC confined to a $100 \mu \mathrm{L}$ spherical depot. c $4 \mathrm{mg}$ TAC dispersed throughout the entire vitreous. Trend curves represent best fits to a first order function (see text).

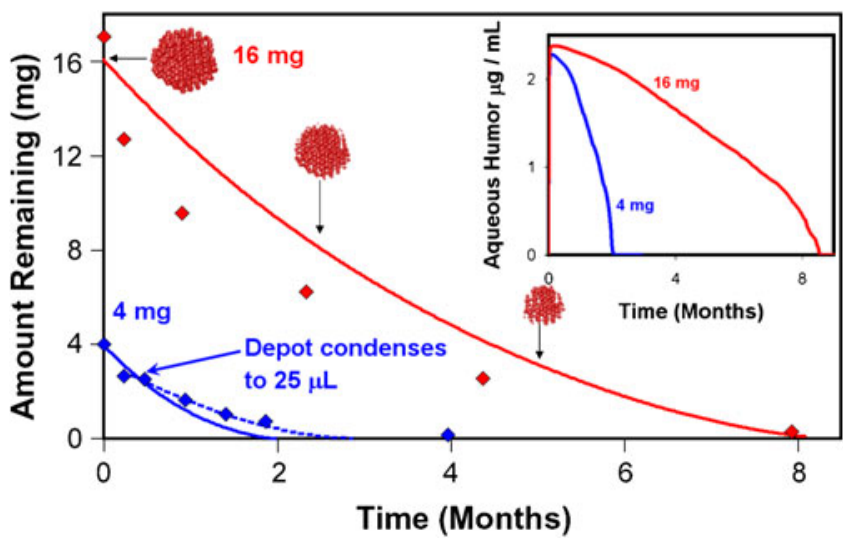

Fig. 12 Dissolution profiles for $4 \mathrm{mg}$ and $16 \mathrm{mg}$ TAC confined to a $100 \mu \mathrm{L}$ spherical vitreous depot compared with experimental data from reference 5. Curves represent results in the limit of infinitesimally small particles. The dashed curve for $4 \mathrm{mg}$ restarts the simulation by distributing $2.7 \mathrm{mg}$ of drug in a $25 \mu \mathrm{L}$ depot to approximate the influence of depot condensation observed in vivo. The inset shows the mean aqueous humor concentrations predicted.

the infinite sinks applied in the anterior tissues of the iris and ciliary body are what produces the low concentrations in the aqueous humor, within experimental error. The experimental value for fluorescein glucuronide exhibits a clearance rate appropriate for a small molecule like sucrose, but the aqueous humor concentration falls somewhat below the line predicted by Eq. 4. This reduced aqueous humor concentration may be due in part to its lipophilicity, which might cause it to partition preferentially into the iris and ciliary body and to be cleared slightly more rapidly than would be accounted for strictly on the basis of aqueous humor production and elimination.

Of the three ocular models, the new geometry presented in Fig. 1 provides the best overall match to the data for materials not cleared by the posterior pathway, both in terms of the elimination rate and the average concentration

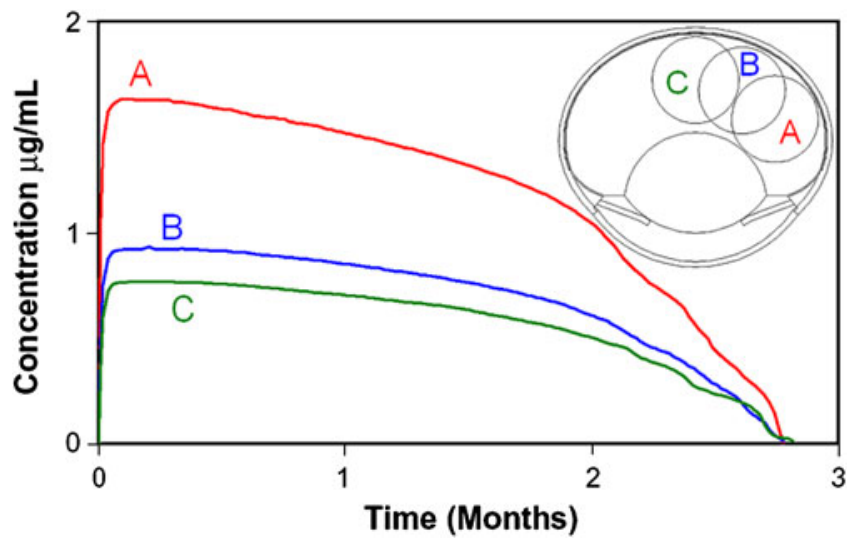

Fig. I3 Dissolution profiles for $4 \mathrm{mg}$ TAC divided amongst nineteen equally sized $(738 \mu \mathrm{m})$ particles confined to a $100 \mu \mathrm{L}$ spherical vitreous depot located in three different regions of the vitreous as shown in the inset. 
ratio. The simulated points fall very close to the data and to the line calculated from Eq. 4. Next in agreement is the completed Friedrich model, which provides a reasonable match to the elimination rate, but which systematically underestimates the aqueous humor concentration by at least a factor of two. The predictions from the Park model significantly underestimate both the clearance rate and the concentration ratio. These results are consistent with the "bottleneck" effect apparent from Fig. 2. In comparison with the Friedrich geometry, the Park model deepened the lens cavity and elongated the eye, which resulted in a narrower band connecting the vitreous and aqueous compartments, leading to the most serious underestimates of clearance rates and aqueous humor concentrations. The more anatomically accurate approach produced a wider eye than the Friedrich geometry, which increased the area of contact between the compartments. The area of contact was further increased by including the gap between the vitreous and ciliary body, which might be an important mechanism contributing to the clearance of these materials. All three models provide comparable and adequate predictions for the clearance and concentration ratio for fluorescein, which falls significantly away from the curve, having a much more rapid clearance and lower aqueous humor concentration.

Park et al. validated the ability for their model to predict the concentration profile within the vitreous at a particular point in time after central bolus injection (4), but did not offer predictions of concentrations in the aqueous humor or the rate of anterior clearance. The original geometry proposed by Friedrich et al. (3) was validated similarly but did not contain the entire aqueous humor compartment and thus could not be used to directly simulate the aqueous humor concentration, though in principle it could be calculated. Models that accurately predict the anterior concentration and clearance rates may be useful for estimating total ocular drug content from measurements of drug levels in the anterior compartment alone. Note that the aqueous humor production rate of the current model is $36 \%$ higher than that used in the previous models, and thus the clearance of materials which exclusively use the anterior pathway would be accelerated by this amount. The influence of aqueous humor production rate on ocular clearance will become less important as the strength of the retinal sink increases. The time to total dissolution for any suspension model would be increased by less than $50,000 \mathrm{~s}$ by reducing the aqueous humor production rate to the lower value.

The simulations in Fig. 6 assumed a normotensive pressure of 15 Torr for each of the models. The effect of intraocular pressure will impact the shape of the clearance curves, as illustrated for the new geometry in Fig. 7. Pressure does not influence clearance of small molecules. But for large, slowly diffusing materials, the clearance is influenced by pressure in a complicated manner. As the diffusivity decreases, the relative contribution of convective flow on transport increases.

Fig. 8a, which demonstrates the validity of the dissolution method for a single particle in a symmetric spherical geometry, compares the exact solution (solid curve) to three other curves. The curve marked "infinitely large vitreous" plots the kinetics for dissolution of the same spherical bead in an infinite expanse. This very well-known calculation, derived by H. P. Hopfenberg (39) more than 30 years ago, was cast in an elegant mathematical form which provides an intuitive comparison to release rates from other shapes such as slabs and cylinders. The exact solution for our current derivation reduces to the Hopfenberg curve for a spherical particle in the limit $\mathrm{R}_{\mathrm{v}} \rightarrow \infty$. An exponential curve projected from the initial time decay of the Hopfenberg solution is also plotted to illustrate the approximate resemblance between the two solutions. The exact solution for the finite vitreous falls much closer to a linear curve at early times, and then begins to resemble the Hopfenberg solution at later times. Curves for the time dependence of the dissolution rate for the various models (the magnitude of the slopes of the curves) appear in Fig. 8b.

The simulations reported here ignore the early changes in the relative locations of particles immediately after dosing. The particle positions are idealized as a perfect hexagonal array confined to a sphere and remain fixed in space throughout the dissolution process. Also not investigated in detail is the transient state at early time, during which the drug concentration distribution achieves a quasisteady-state geometric distribution. The simulations can actually be conducted with sufficient accuracy to resolve the finer points of the time dependence after immediate placement. If one calculates the flux through a surface bounding the particles, the peak flux through this surface is established within an hour. Within about 1 day it settles down to the quasi-steady-state value. The peak flux at 1 hour exceeds the quasi-steady-state flux by only about $25 \%$. What was ignored that is perhaps more significant is the fact that the solution phase of the injection would have been saturated with dissolved drug. This would actually have suppressed the initial rate of drug dissolution until the initial bolus of dissolved drug would have diffused away from the injection site. But all of these approximations are immaterial to the main emphasis of this investigation, to determine the influence of particle size on the dissolution of a suspension depot in the vitreous.

A surprising and perhaps somewhat nonintuitive observation is that the dissolution versus time profiles for the various suspension models plotted in Figs. 10 and 11 become independent of particle size as the diameter decreases below a certain value, which depends upon the type of depot being simulated. This does not follow the trend expected from the 
classical pharmaceutical studies on the impact of particle size on dissolution of suspensions of finely divided particles (4042). In those studies, which were designed with a view towards oral administration, the dissolution situation involves the rapid stirring of a suspension aliquot in a relatively large volume of release medium, such as the USP Method Type II. In such a method, the exposed surface area of each particle is more or less uniformly accessible to the release medium, which is the situation of ingested drug in the stomach after oral administration. But the situation of an intravitreal depot is quite different. The dissolution of particles in the interior of the depot is shielded from dissolution compared to those near the exterior; thus, each particle is not uniformly accessible to the same conditions throughout the dissolution process.

Since the particle size independent limits are so much greater than the sizes of the particles in either product, it is justified to use the size-independent predictions to compare simulation results with the in vivo experimental results in Fig. 12. Each simulation provides a fair match to the trend illustrated by the data for the 4 and $16 \mathrm{mg}$ spherical depots, although the $4 \mathrm{mg}$ simulation predicts a somewhat more rapid dissolution rate than that observed in vivo. Kim et al. observed (5) that after injection, the depot does not remain stationary, but appears to settle under the influence of gravity. Thus, it is conceivable that the interparticle spacing does not remain constant with time, but rather that the entire depot might be condensing after injection. Since it would be difficult to simulate a dissolution process in which the particle locations would be changing with time, the more crude approach described in the Results section was used, redistributing the mass remaining after 10 days from the initial $100 \mu \mathrm{L}$ depot into a smaller $25 \mu \mathrm{L}$ spherical depot. This second curve provides a better fit to the data at later time, extending the suspension duration to nearly 3 months. The data suggest that further reductions in the depot volume might be occurring with time.

The predicted profiles for the aqueous humor concentration appear in the inset of Fig. 12. The peak aqueous humor concentration appearing in the inset of Fig. 12 for both the $4 \mathrm{mg}$ and $16 \mathrm{mg}$ suspensions are quite similar, about $2.3 \mu \mathrm{g} / \mathrm{mL}$. The aqueous humor concentrations were not reported from the study of Kim et al. (5). However, the values are quite similar to the concentrations reported in other studies $(34,43)$. For example, Beer et al. (34) reported peak aqueous humor concentrations of $2-7 \mu \mathrm{g} / \mathrm{mL}$ following injection of $4 \mathrm{mg}$ KENALOG®-40 in human subjects. The high value reported by Beer et al. was for the case of a patient that had undergone vitrectomy; thus, it is not clear whether or not the same type of depot would have formed upon intravitreal injection into such an eye. The lower values measured in vivo were more in line with the current predictions. The results of Fig. 13 indicate that the depot location could have an important impact upon the aqueous humor concentrations. The anterior elimination pathway constitutes a greater fraction of the overall elimination as the depot resides closer to the hyaloid membrane.

Simulations of both types have been conducted in an anatomically accurate human eye model. The vitreous volume increases from $1.7 \mathrm{~mL}$ for the rabbit to nearly $5 \mathrm{~mL}$ for the human. The volume fraction occupied by the lens is smaller in the human ocular anatomy, and it is placed more anteriorly in proportion to the rabbit eye. The slanted line in Fig. 6 predicted using Eq. 4 for the human eye model would be parallel to the line for the rabbit eye model, but a factor of more than three-fold higher. However, the same material injected into the two species will be eliminated more slowly from the human eye, since the time decay for diffusion out of a sphere varies inversely proportional to the square of the radius (44). Since the aqueous humor production rate is also slightly smaller in the human eye, $2.4 \mu \mathrm{L} / \mathrm{min}$ (45), the net prediction is that clearance out of the human eye should proceed at about $40 \%$ of the clearance rate for the rabbit eye. This makes the prediction for the aqueous/vitreous humor concentration ratio comparable for the same material injected in the two species. Preliminary simulations of materials injected into the human eye allowing only for elimination through the anterior pathway confirm the predictions above (not shown). Simulations of the dissolution of TAC suspension depots also allowing for an infinite sink in the retina predicted dissolution rates that were also quite similar to those predicted in the rabbit eye model, with similar independence of dissolution rate and dependence of aqueous humor concentration on depot position.

\section{CONCLUSIONS}

A computational method was developed for simulating dissolution and drug delivery from a suspension of spheres placed in the vitreous. The method was capable of predicting the time dependence of the boundary between solid and dissolved drug for each individual particle and was validated against an analytical solution for a single spherical particle placed in the center of an idealized spherical eye model. Particle depots of varying configurations were located in the vitreous of an anatomically accurate model of the rabbit eye. This model was separately validated by demonstrating its superior ability to predict the clearance rates of dissolved materials after intravitreal injection, compared to two other models in the literature.

Since the particle diameters below which dissolution rate ceased to increase for the various depot types are much larger than the particle sizes for either KENALOG ${ }^{\circledR}-40$ $(14-21 \mu \mathrm{M})$ or TRIESENCETM $(5-6 \mu \mathrm{M})$, it is anticipated 
that the dissolution rates for these two products would be comparable under conditions in which they form welldefined depots within the vitreous. The credibility of these simulations is supported by the fact that the dissolution rate predicted in the limit of infinitesimally small particle size matches the rate observed in vivo (5).

These simulation studies predict that KENALOG ${ }^{\circledR}-40$ and TRIESENCETM, when injected into a well-formed vitreous, such that a depot is formed, should have quite similar delivery profiles. As a note added in proof, Robinson et al. reported nearly identical vitreous half-lives for KENALOG®-40 and for their version of a preservative-free Triamcinolone Acetonide formulation (46). The drug particle size had been reduced to about $4 \mu \mathrm{m}$ by micronization for the preservative-free formulation (5), whereas they noted a mean particle size of $17.3 \mu \mathrm{m}$ for the lot of KENALOG ${ }^{\circledR}-40$ used in their study in NZW rabbits (46). Thus, the disparity in particle size between KENALOG®-40 and their preservative-free product is similar to what Alcon has measured between KENALOG ${ }^{\circledR}-40$ and TRIESENGE ${ }^{\mathrm{TM}}$.

For the future, the current modeling approach can be improved to incorporate:

- More details regarding the nature, dimensions, and species variation of the retrozonular space of Petit as they become available.

- Multiphase fluid modeling of the injection process (47), possibly treating the injected phase as a particle suspension.

- Insights from the tissue biomechanics of infusion processes in other regions of the body (48).

- The effects of vitreous gel syneresis upon aging (2) and vitrectomy (49).

\section{ACKNOWLEDGMENTS}

I am indebted to David Maurice, who, through our personal correspondence in 2000, shared with me an unpublished manuscript apprising me of the significance of the bottleneck effect of the ocular geometry.

Open Access This article is distributed under the terms of the Creative Commons Attribution Noncommercial License which permits any noncommercial use, distribution, and reproduction in any medium, provided the original author(s) and source are credited.

\section{APPENDIX}

The purpose of this appendix is to calculate the dissolution rate of a bead of initial radius $\mathrm{R}_{0}$ placed in the center of a vitreous sphere of radius $R_{v}$, having vitreous diffusivity $D$, and an infinite sink applied at the outer spherical vitreous surface (see Fig. 14). We are not interested in the details of the early time dependence, but will calculate the rate after the initial distribution of drug is established within the vitreous and ignore the loss of drug required to establish this initial distribution. We begin by first deriving the spatial distribution of drug in the vitreous after establishing the quasi-steady state. The applicable equation within the vitreous sphere is

$\frac{\mathrm{D}}{\mathrm{r}^{2}} \frac{\mathrm{d}}{\mathrm{dr}}\left(\mathrm{r}^{2} \frac{\mathrm{dC}}{\mathrm{dr}}\right)=0$

The solution to this equation is:

$\mathrm{C}(\mathrm{r})=\frac{\mathrm{E}}{\mathrm{r}}+\mathrm{F}$

We apply the value boundary conditions; at the particle surface, at radius $\mathrm{R}$, the drug is fixed at the solubility limit $\mathrm{S}$; and at the outer vitreous, at radius $\mathrm{R}_{\mathrm{v}}$, the drug concentration is set to zero. This enables us to derive expressions for the coefficients $\mathrm{E}$ and $\mathrm{F}$ in Eq. A-2:

$\mathrm{C}(\mathrm{R})=\mathrm{S}=\frac{\mathrm{E}}{\mathrm{R}}+\mathrm{F}$

$\mathrm{G}\left(\mathrm{R}_{\mathrm{v}}\right)=0=\frac{\mathrm{E}}{\mathrm{R}_{\mathrm{v}}}+\mathrm{F}$

From Eq. A-4,

$\mathrm{F}=-\frac{\mathrm{E}}{\mathrm{R}_{\mathrm{v}}}$

Substituting Eq. A-5 into Eq. A-3,

$\mathrm{S}=\frac{\mathrm{E}}{\mathrm{R}}-\frac{\mathrm{E}}{\mathrm{R}_{\mathrm{V}}}=\mathrm{E}\left(\frac{1}{\mathrm{R}}-\frac{1}{\mathrm{R}_{\mathrm{V}}}\right)$

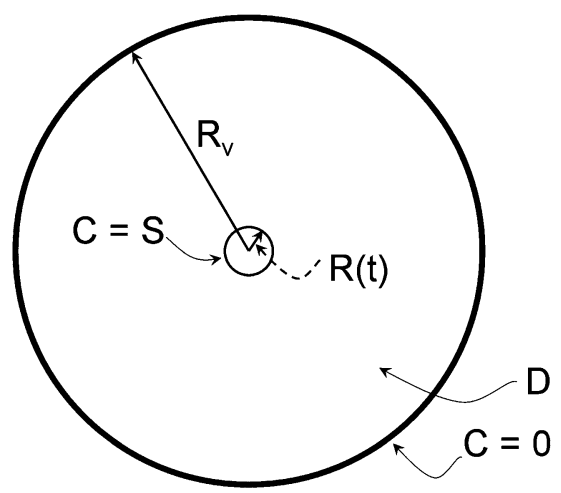

Fig. I4 Diagram for the reference calculation in the Appendix, a spherical drug bead dissolving in the center of an idealized spherical vitreous domain bounded by an infinite sink. 
from which we obtain an expression for $\mathrm{E}$ :

$\mathrm{E}=\frac{\mathrm{S}}{\left(\frac{1}{\mathrm{R}}-\frac{1}{\mathrm{R}_{\mathrm{v}}}\right)}$

Substituting Eqs. A-5 and A-6 into Eq. A-3, we obtain an expression for the spatial distribution of drug in the vitreous:

$\mathrm{C}(\mathrm{r})=\frac{\mathrm{S}}{\left(\frac{1}{\mathrm{R}}-\frac{1}{\mathrm{R}_{\mathrm{v}}}\right)}\left[\frac{1}{\mathrm{r}}-\frac{1}{\mathrm{R}_{\mathrm{v}}}\right]$

The flux of drug through the surface at $r=\mathrm{R}$ is given by:

Flux $\left.\right|_{\mathrm{r}=\mathrm{R}}=-\left.\mathrm{D} \frac{\mathrm{dC}}{\mathrm{dr}}\right|_{\mathrm{r}=\mathrm{R}}=+\frac{1}{\mathrm{R}^{2}} \frac{\mathrm{D} \mathrm{S}}{\left(\frac{1}{\mathrm{R}}-\frac{1}{\mathrm{R}_{\mathrm{v}}}\right)}$

The rate of mass loss from the bead is obtained by integrating this flux over the entire surface:

$\frac{\mathrm{dM}}{\mathrm{dt}}=4 \pi \mathrm{R}^{2 *}$ Flux $\left.\right|_{\mathrm{r}=\mathrm{R}}=\frac{4 \pi \mathrm{DS}}{\left(\frac{1}{\mathrm{R}}-\frac{1}{\mathrm{R}_{\mathrm{v}}}\right)}$

Now consider a shell of thickness $\delta R$ on the exterior of the spherical bead. The mass of solid undissolved drug in this shell is:

$\delta \mathrm{M}=-4 \pi \mathrm{R}^{2} \rho \delta \mathrm{R}$

where $\rho$ is the density of undissolved drug. From Eq. A-10 we obtain the rate of change in mass of the bead with change in radius:

$\frac{\mathrm{dM}}{\mathrm{dR}}=-4 \pi \mathrm{R}^{2} \rho$

Note the minus sign appears in Eqs. A-10, A-11 because mass is lost during dissolution. Using the chain rule,

$\frac{\mathrm{dM}}{\mathrm{dt}}=\frac{\mathrm{dM}}{\mathrm{dR}} \frac{\mathrm{dR}}{\mathrm{dt}}$

Rearranging Eq. A-12 and substituting in from Eqs. A-9, A-11, we obtain an expression for the rate in change of $\mathrm{R}$ with time:

$$
\begin{aligned}
\frac{\mathrm{dR}}{\mathrm{dt}} & =\frac{\mathrm{dM}}{\mathrm{dt}} / \frac{\mathrm{dM}}{\mathrm{dR}}=\frac{4 \pi \mathrm{DS}}{\left(\frac{1}{\mathrm{R}}-\frac{1}{\mathrm{R}_{\mathrm{v}}}\right)} /\left(-4 \pi \mathrm{R}^{2} \rho\right) \\
& =\frac{-\mathrm{DS}}{\mathrm{R}^{2} \rho\left(\frac{1}{\mathrm{R}}-\frac{1}{\mathrm{R}_{\mathrm{v}}}\right)}=\frac{-\mathrm{DS}}{\rho\left(\mathrm{R}-\frac{\mathrm{R}^{2}}{\mathrm{R}_{\mathrm{v}}}\right)}
\end{aligned}
$$

Rearranging this equation, we obtain an expression which is totally separated in $\mathrm{R}$ and $\mathrm{t}$ :

$\mathrm{dR}\left(\mathrm{R}-\frac{\mathrm{R}^{2}}{\mathrm{R}_{\mathrm{v}}}\right)=\frac{-\mathrm{DS}}{\rho} \mathrm{dt}$
Integrating this expression on both sides,

$\int\left(\mathrm{R}-\frac{\mathrm{R}^{2}}{\mathrm{R}_{\mathrm{v}}}\right) \mathrm{dR}=\frac{-\mathrm{DS}}{\rho} \int \mathrm{dt}$

which leads to

$-\frac{\rho}{\mathrm{DS}}\left[\frac{\mathrm{R}^{2}}{2}-\frac{\mathrm{R}^{3}}{3 \mathrm{R}_{\mathrm{v}}}\right]=+\mathrm{t}+\mathrm{K}$

where $\mathrm{K}$ is a constant of integration. At $t=0, R=\mathrm{R}_{0}$, from which we determine the expression for $\mathrm{K}$ :

$\mathrm{K}=-\frac{\rho}{\mathrm{DS}}\left[\frac{\mathrm{R}_{0}^{2}}{2}-\frac{\mathrm{R}_{0}^{3}}{3 \mathrm{R}_{\mathrm{v}}}\right]$

Thus, the exact expression for the dependence of the particle radius upon time may be expressed in terms of $t$ as a function of $\mathrm{R}$ as follows:

$\mathrm{t}=\frac{\rho}{\mathrm{DS}}\left\{\left[\frac{\mathrm{R}_{0}{ }^{2}}{2}-\frac{\mathrm{R}_{0}{ }^{3}}{3 \mathrm{R}_{\mathrm{v}}}\right]-\left[\frac{\mathrm{R}^{2}}{2}-\frac{\mathrm{R}^{3}}{3 \mathrm{R}_{\mathrm{v}}}\right]\right\}$

Admittedly, this is not exactly in the form that we desired; instead of an expression of $\mathrm{R}$ in terms of $\mathrm{t}$, we have a function of $\mathrm{t}$ in terms of $\mathrm{R}$. But it is an extremely wellbehaved function which was used to calculate the solid curve depicted in Fig. 8a. An instructive result is obtained by taking the limit of this expression as $\mathrm{R}_{\mathrm{v}} \rightarrow \infty$ :

$\mathrm{t} \underset{\operatorname{Lim} \mathrm{R}_{\mathrm{v}} \rightarrow \infty}{\rightarrow} \frac{\rho}{2 \mathrm{DS}}\left(\mathrm{R}_{0}^{2}-\mathrm{R}^{2}\right)$

From this we can derive an approximate expression for $\mathrm{R}$ in terms of $\mathrm{t}$ :

$\mathrm{R} \approx \mathrm{R}_{0} \sqrt{1-\frac{2 \mathrm{DS}}{\mathrm{R}_{0}^{2} \rho} t}$

The Taylor series expansion of Eq. A-20 is

$\mathrm{R} \approx \mathrm{R}_{0}-\frac{\mathrm{DS}}{\mathrm{R}_{0} \rho} \mathrm{t}-\frac{\mathrm{D}^{2} \mathrm{~S}^{2}}{2 \mathrm{R}_{0}{ }^{3} \rho^{2}} \mathrm{t}^{2}+\ldots$

If we limit the Taylor series expansion in Eq. A-21 to the first-order term, we find that the drug bead radius varies linearly with time. This is the same dependence predicted by Hopfenberg (35) for the case of a spherical bead dissolving in an infinite expanse. It is noteworthy that the dissolution rate (solid curve, Fig. 8b) falls in between constant, zero-order release and first-order release (exponential decay). The dissolution rate exceeds the first-order dependence until the drug bead is nearly fully exhausted, and then the dissolution process decreases rapidly and stops suddenly. The time at which the dissolution is complete can be calculated by evaluating Eq. A-18 in the limit when $\mathrm{R}(\mathrm{t})$ becomes zero:

$\mathrm{t}_{\mathrm{end}}=\frac{\rho}{\mathrm{DS}}\left[\frac{\mathrm{R}_{0}{ }^{2}}{2}-\frac{\mathrm{R}_{0}{ }^{3}}{3 \mathrm{R}_{\mathrm{v}}}\right]$

$(\mathrm{A}-22)$ 


\section{REFERENCES}

1. Sawada T, Nakamura J, Nishida Y, Kani K, Morikawa S, Inubushi T. Magnetic resonance imaging studies of the volume of the rabbit eye with intravenous mannitol. Curr Eye Res. 2002;25:173-7.

2. Sebag J. The vitreous: structure, function and pathobiology. New York: Springer-Verlag; 1989.

3. Friedrich S, Cheng Y-L, Saville B. Finite element modeling of drug distribution in the vitreous humor of the rabbit eye. Ann Biomed Eng. 1997;25:303-14.

4. Park J, Bungay PM, Lutz RJ, Augsburger JJ, Millard RW, Roy AS, et al. Evaluation of coupled convective-diffusive transport of drugs administered by intravitreal injection and controlled release implant. J Control Release 2005;105:279-95.

5. Kim H, Gsaky KG, Gravlin L. Safety and pharmacokinetics of a preservative-free Triamcinolone Acetonide formulation for intravitreal administration. Retina 2006;26:523-30.

6. Heys JJ, Barocas VH, Taravella MJ. Modeling passive mechanical interaction between aqueous humor and iris. Trans ASME. 2001;123:540-7.

7. Heys JJ, Barocas VH. Computational evaluation of the role of accommodation in pigmentary glaucoma. Invest Ophthalmol Vis Sci. 2002;43:700-8.

8. Wu JC, Wong EC, Arrindell EL, Simons KB, Jesmanowicz A, Hyde JS. In vivo determination of anisotropic diffusion of water and the $\mathrm{T} 1$ and $\mathrm{T} 2$ times in the rabbit lens by high-resolution magnetic resonance imaging. Invest Ophthalmol Vis Sci. 1993;34:2151-8.

9. Bron AJ, Tripathi RC, Tripathi BJ. Wolff's Anatomy of the Eye and Orbit, $8^{\text {th }}$ edition. Chapter 6: The eyeball and its dimensions; Chapter 8: The posterior chamber and ciliary body. Oxford University Press, New York, 1997.

10. Missel PJ. Finite and infinitesimal representations of the vasculature: ocular drug clearance by vascular and hydraulic effects. Ann Biomed Eng. 2002;30:1128-39.

11. Missel PJ. Hydraulic flow and vascular clearance influences on intravitreal drug delivery. Pharm Res. 2002;19:1636-47.

12. Faber TE. Fluid dynamics for physicists. Cambridge: Cambridge University Press; 1995. p. 238.

13. Tritton DJ. Physical fluid dynamics. Oxford: Oxford University Press; 1988. p. 52-3.

14. Reitsamer HA, Kiel JW. Relationship between ciliary blood flow and aqueous production in rabbits. Invest Ophthalmol Vis Sci. 2003;44:3967-71.

15. Reitsamer HA, Bogner B, Tockner B, Kiel JW. Effects of Dorzolamide on choroidal blood flow, ciliary blood flow, and aqueous production in rabbits. Invest Ophthalmol Vis Sci. 2009;50:2301-7.

16. Kinsey VE, Reddy DVN. Chemistry and dynamics of aqueous humor. In: Prince JH, editor. The rabbit eye in research. Springfield: C.C. Thomas; 1964. p. 218-319.

17. Blondeau P, Tetrault JP, Papamarkakis C. Diurnal variation of episcleral venouspressure in healthy patients: a pilot study. J Glaucoma. 2001;10:18-24.

18. Funk RH, Gehr J, Rohen JW. Short-term hemodynamic changes in episcleral arteriovenous anastomoses correlate with venous pressure and IOP changes in the albino rabbit. Curr Eye Res. 1996;15:87-93.

19. Krupin T, Rosenberg LF, Sandridge AL, Bock CJ, Berman A, Ruderman JM. Effects of topical k-strophanthin on aqueous humor and corneal dynamics. J Glaucoma. 1995;4:327-33.

20. Xu J, Heys JJ, Barocas VH, Randolph TW. Permeability and diffusion in vitreous humor: implications for drug delivery. Pharm Res. 2000;17:664-9.
21. Fatt I, Hedbys B. Flow of water in the sclera. Exp Eye Res. 1970;10:243-9.

22. Wiedmann TS, Bhatia R, Wattenberg LW. J Control Release. 2000;65:43-7.

23. Kaiser RJ, Maurice DM. The diffusion of fluorescein in the lens. Exp Eye Res. 1964;3:156-65.

24. Maurice DM. Protein dynamics in the eye studied with labeled proteins. Am J Ophthalmol. 1959;47:361-7.

25. Araie M, Maurice DM. The loss of fluorescein, fluorescein glucuronide and fluorescein isothiocyanate dextran from the vitreous by the anterior and retinal pathways. Exp Eye Res. 1991;52:27-39.

26. Maurice DM. Flow of water between aqueous and vitreous compartments in the rabbit eye. Am J Physiol. 1987;252:F104108.

27. Maurice DM. Injection of drugs into the vitreous body. In: Leopold T, Burns R, editors. Symposium on ocular therapy, vol. 9. London: Wiley; 1976. p. 59-72.

28. Weiner AL, Missel PJ, Chastain JE, Yaacobi Y, Mitra AK, Kompella UB, et al. Administration of anecortave acetate in a finite element physiologic ocular pharmacokinetic model. Invest Ophthalmol Vis Sci 47:ARVO E-Abstract 5084 (2006).

29. Prausnitz MR, Noonan JS. Permeability of cornea, sclera, and conjunctiva: a literature analysis for drug delivery to the eye. J Pharm Sci. 1998;87:1479-88.

30. Olsen TW, Edelhauser HF, Lim JI, Geroski DH. Human scleral permeability: effects of age, cryotherapy, transscleral diode laser, and surgical thinning. Invest Ophthalmol Vis Sci. 1995;36:1893903.

31. Bito LZ, Salvador EV. Intraocular fluid dynamics III. The site and mechanism of prostaglandin transfer across the blood ocular fluid barriers. Exp Eye Res. 1972;14:233-41.

32. Johnson F, Maurice D. A simple method of measuring aqueous humor flow with intravitreal fluoresceinated dextrans. Exp Eye Res. 1984;39:791-805.

33. Seto C, Araie M, Takase M. Study of fluorescein glucuronide. II. A comparative ocular kinetic study of fluorescein and fluorescein glucuronide. Graefes Arch Clin Exp Ophthalmol. 1986;224:1137 .

34. Beer PM, Bakri SJ, Singh RJ, Liu W, Peters III GB, Miller M. Intraocular concentration and pharmacokinetics of Triamcinolone Acetonide after a single intravitreal injection. Ophthalmol. 2003;1 10:681-6.

35. Barton KA, Shui Y-B, Petrash M, Beeb DC. Comment on: Stokes-Einstein equation and the physiologic effects of vitreous surgery. Acta Ophthalmologica Scand. 339-340 (2007).

36. Peeters L, Sanders NN, Braeckmans K, Boussery K, Van de Voorde J, De Smedt SG, et al. Vitreous: a barrier to nonviral ocular gene therapy. Invest Ophthalmol Vis Sci. 2005;46:355361.

37. Missel P, Chastain J, Mitra A, Kompella U, Kansara V, Duvvuri $\mathrm{S}$, et al. In vitro transport and partitioning of AL-4940, active metabolite of angiostatic agent anecortave acetate, in ocular tissues of the posterior segment. J Ocular Pharm Ther. 2010;26:137-145

38. Missel PJ, Lang JC, Rodeheaver DP, Jani R, Chowhan MA, Dagnon T. Design and evaluation of ophthalmic pharmaceutical products. In Florence AT, Siepman J (eds), Modern Pharmaceutics, fifth Edition, Informa Healthcare, New York, 2009, Volume 2, p. 142.

39. Hopfenberg HP. Controlled release from erodible slabs, cylinders, and spheres. In: Paul DR, Harris FW, editors. Controlled release from polymeric formulations, ACS Symposium Series 33. Washington, D.C.: American Chemical Society; 1976. p. 26-31. 
40. Higuchi WI, Hiestand EN. Dissolution rates of finely divided drug powders I. Effect of a distribution of particle sizes in a diffusioncontrolled process. J Pharm Sci. 1963;52:67-71.

41. Mauger JW, Howard SA, Amin K. Dissolution profiles for finely divided drug suspensions. J Pharm Sci. 1983;72:190-3.

42. Pereira de Almeida L, Simoes S, Brito P, Portugal A, Figueiredo M. Modeling dissolution of sparingly soluble multisized powders. J Pharm Sci. 1997;86:725-32.

43. Banz K, Rodger J, Carroll S, Boyd B. Slow release formulations of Triamcinolone Acetonide (TA) for intra-vitreal injection provide sustained TA concentrations in a rabbit pharmacokinetic model. ARVO E-Abstract 5808 (2007).

44. Crank J. The mathematics of diffusion. 2nd ed. Oxford: Clarendon; 1975. chapter 6.

45. Hart WM. Adler's physiology of the eye. St. Louis: Mosby Year Book; 1992. p. 888.
46. Robinson MR, Kim H, Gravlin L, Tansey G, Grimes G, Potti G, et al. Preclinical evaluation of a triamcinolone acetonide preservative free (TAC-PF) formulation for intravitreal injection. Invest Ophthalmol Vis Sci 45:ARVO E-Abstract 5058, 2004.

47. Lin H-H. Finite element modeling of drug transport processes after an intravitreal injection - a study of the effects of drug-phase geometry on bioavailability and toxicity. Masters Thesis, Department of Chemical Engineering and Applied Chemistry, University of Toronto (1997).

48. Morrison PF, Chen MY, Chadwick RS, Lonser RR, Oldfield EH. Focal delivery during direct infusion to brain: role of flow rate, catheter diameter, and tissue mechanics. Am J Physiol Regul Integr Comp Physiol. 1999;277:1218-29.

49. Lee SS, Ghosn C, Yu Z, Zacharias LC, Kao H, Lanni C, et al. Vitreous VEGF clearance is increased after vitrectomy. Invest Ophthalmol Vis Sci. 2010;51:2135-8. 